\title{
BMJ Open Protective potential of high-intensity interval training on cardiac structure and function after COVID-19: protocol and statistical analysis plan for an investigator-blinded randomised controlled trial
}

Iben Elmerdahl Rasmussen (D) , ${ }^{1}$ Frederik Foged, ${ }^{1}$ Josephine Bjørn Budde, ${ }^{1,2}$ Rasmus Syberg Rasmussen (1) , ' Villads Rasmussen, ${ }^{1}$ Mark Lyngbæk, ${ }^{1}$ Simon Jønck, ${ }^{1}$ Rikke Krogh-Madsen, ${ }^{1,3}$ Birgitte Lindegaard, ${ }^{1,4}$ Mathias Ried-Larsen, ${ }^{1}$ Peter Godsk Jørgensen, ${ }^{5}$ Morten Asp Vonsild Lund, ${ }^{2,5}$ Lars Køber, ${ }^{5}$ Niels Vejlstrup, ${ }^{5}$ Bente Klarlund Pedersen, ${ }^{1}$ Ronan M G Berg (D) , 1,2,6,7 Regitse Højgaard Christensen ${ }^{1,5}$

To cite: Rasmussen IE, Foged F, Bjørn Budde J, et al. Protective potential of highintensity interval training on cardiac structure and function after COVID-19: protocol and statistical analysis plan for an investigator-blinded randomised controlled trial. BMJ Open 2021;11:e048281. doi:10.1136/ bmjopen-2020-048281

- Prepublication history and additional supplemental material for this paper are available online. To view these files, please visit the journal online (http://dx.doi.org/10.1136/ bmjopen-2020-048281).

IER and FF contributed equally.

Received 05 January 2021 Accepted 13 October 2021

Check for updates

(c) Author(s) (or their employer(s)) 2021. Re-use permitted under CC BY-NC. No commercial re-use. See rights and permissions. Published by BMJ.

For numbered affiliations see end of article.

\section{Correspondence to}

Dr Regitse Højgaard

Christensen;

regitse.hoejgaard.christensen@ regionh.dk

\section{ABSTRACT}

Introduction COVID-19 is associated with a marked systemic inflammatory response with concomitant cardiac injury and remodelling, but it is currently unknown whether the latter is reversible. Given that high-intensity interval training (HIIT) is a powerful stimulus to improve cardiorespiratory fitness while also eliciting marked anti-inflammatory effects, it may be an important countermeasure of reducing cardiopulmonary morbidity following COVID-19.

Methods and analysis 40 COVID-19 survivors who have been discharged from hospital will be included in this investigator-blinded randomised study with a 12-week HIIT intervention. Patients will be 1:1 block-randomised by sex to either a supervised HIIT exercise group or standard care (control group). The main hypothesis is that a 12week HIIT scheme is a safe way to improve loss of cardiac mass and associated cardiorespiratory fitness, despite hypothesised limited HIIT-induced changes in conventional lung function indices per se. Ultimately, we hypothesise that the HIIT scheme will reduce post-COVID-19 symptoms and improve quality of life.

Ethics and dissemination This study is approved by the Scientific Ethical Committee at the Capital Region of Denmark (H-20033733, including amendments 75068 and 75799) and registered at ClinicalTrials.gov (NCT04647734, pre-results). The findings will be published in a peerreviewed journal, including cases of positive, negative and inconclusive results.

Trial registration number NCT04549337.

\section{INTRODUCTION}

The exaggerated systemic proinflammatory response, often coined as a 'cytokine storm', has been hypothesised to account for the now

\section{Strengths and limitations of this study}

- This is the first randomised study to examine the safety and effects of supervised high-intensity interval training (HIIT) on cardiac structure and function after COVID-19.

- A 12-week HIIT scheme consisting of three weekly sessions of $38 \mathrm{~min}$ has previously shown to mitigate comorbidities that are like those seen in COVID-19 survivors.

- Changes in cardiopulmonary function and exercise capacity are assessed by state-of-the-art methods.

- We only include previously hospitalised patients, which limits the generalisability of the study and introduces a possible selection bias.

- Various measures are only measured after COVID-19 infection; the baseline prior to infection is thus unknown.

well-documented association of COVID-19 with cardiopulmonary complications such asmyocardial injury, ${ }^{1-4}$ especially in people with high cardiometabolic risk. ${ }^{5}$ It is currently unknown whether COVID-19-associated cardiopulmonary changes are irreversible, but it has been documented that COVID-19 survivors are at an increased risk of cardiopulmonary morbidity and mortality ${ }^{6}$ and chronic physical disability. ${ }^{7}$ An additional important contributing factor in this context is the prolonged bed-rest associated with hospital admission for COVID-19. ${ }^{8}$ It is well known that bed-rest and physical inactivity of only a few weeks in the general population can increase 
muscle wasting, induce insulin resistance, induce cardiac atrophy and cause a substantial decline in cardiorespiratory fitness as assessed by maximal oxygen consumption $\left(\mathrm{VO}_{2} \max \right){ }^{9-13}$ The prolonged bed-rest combined with hyperinflammation may cause greater impaired cardiopulmonary function and contribute to reduced cardiorespiratory fitness and persistent and often invalidating symptoms, including dyspnoea, chest pain, tachycardia, exercise intolerance and extreme fatigue, to mention a few. ${ }^{14}{ }^{15}$ In addition to physical impairment, patients also suffer from depression and anxiety, which can contribute to reduced quality of life. ${ }^{16}$ The cardiopulmonary consequences of the interaction of extreme hyperinflammation combined with prolonged bed-rest in patients hospitalised with COVID-19 remain to be elucidated.

Exercise training is not only a powerful stimulus for fitness improvement, but also counteracts muscle wasting, improves glucose homeostasis ${ }^{17}$ and elicits a strong anti-inflammatory response. ${ }^{18}$ Previously, a randomised trial on severe acute respiratory syndrome survivors documented that 6 weeks of supervised training significantly increased fitness level $\left(\mathrm{VO}_{2} \max \right)$ by $3.6 \mathrm{~mL} /$ $\mathrm{kg} / \mathrm{min}$ vs $1.0 \mathrm{~mL} / \mathrm{kg} / \mathrm{min}$ in trained versus untrained patients, which corresponds to an increase in fitness level by $10.3 \%$ vs $2.6 \% .{ }^{19}$ As of now, only one study on cardiopulmonary rehabilitation in COVID-19 survivors has been published, which reported that up to 4 weeks of supervised endurance and strength training immediately following hospital discharge was safe, feasible and effective for increasing $\mathrm{VO}_{2} \max$ in a retrospective analysis of 28 patients. ${ }^{20}$ One of the underlying physiological adaptations for the exercise-induced increase in $\mathrm{VO}_{2} \max$ is cardiac hypertrophy, which should be distinguished from pathological hypertrophy, as it is not associated with adverse cardiac events. ${ }^{21} 22$ Other exercise adaptations include increased skeletal muscle mass and muscle respiratory function, while pulmonary exercise adaptations are known to be limited. ${ }^{23}$ Therefore, it remains to be established whether exercise training in patients surviving COVID-19 improves cardiac mass and function, despite anticipated limited effects on pulmonary function. If so, exercise training could potentially restore cardiorespiratory fitness and alleviate the substantial morbidity associated with COVID-19.

There is a growing body of evidence supporting that high-intensity interval training (HIIT) is both an efficacious and safe intervention for targeted cardiopulmonary rehabilitation in a wide array of patient groups. ${ }^{24}$ Hence, several studies have found that HIIT promotes greater health-related benefits, including cardiac function, compared with traditional moderate-intensity training. ${ }^{25}{ }^{26}$ COVID-19 survivors commonly exhibit a marked reduction in pulmonary diffusing capacity, ${ }^{27}$ which is theoretically associated with a concomitant risk of exercise-induced desaturation during exercise, particularly during longer intervals, ${ }^{28}$ which may potentially limit the tolerability and fidelity of HIIT in this patient group. Nonetheless, given that HIIT is implemented in a manner that is tolerable to the individual patient, it may be a potential beneficiary rehabilitation treatment for patients with COVID-19 pneumonia after hospital discharge.

\section{Study aims and hypotheses \\ Primary aim}

The primary aim is to investigate whether HIIT improves structural and functional cardiac and/or pulmonary changes in patients previously hospitalised with COVID19 , thereby improving cardiorespiratory fitness.

\section{Secondary aim}

The secondary aim is to investigate whether HIIT improves post-COVID-19 symptoms and improves quality of life in previously hospitalised COVID-19 survivors.

\section{Primary hypothesis}

We hypothesise that regular supervised HIIT improves cardiac structure and function in patients surviving COVID-19, assessed by cardiac MRI and transthoracic echocardiography (TTE), despite limited or absent HIIT-induced differences between groups in spirometric measures of lung function and pulmonary diffusing capacity.

\section{Secondary hypotheses}

HIIT-induced changes in cardiac structure and function are associated with a reduction in post-COVID-19 symptoms (dyspnoea, chest pain, exercise intolerance, fatigue, palpitations, dizziness, headache and quality of life) and an improved quality of life.

\section{METHODS AND ANALYSIS \\ Overall study design}

The study will be an investigator-blinded randomised controlled trial. Patients $(n=40)$ will be included in the study in relation to their hospital discharge following COVID-19 and will be randomised to 12 weeks of exercise training or standard care as control. Two test days at baseline and postintervention, including a 1-year follow-up, are planned to assess cardiopulmonary fitness, cardiac structure and function, lung function, symptom scores, and quality of life. The enrolment period is planned to start by 1 January 2021, and the study (including 1-year follow-up) is expected to be completed by 31 December 2022. Recruitment for the study will be terminated 1 year after start of enrolment. Due to the risk of low recruitment rate because of a low number of patients hospitalised with SARS-CoV-2 in Denmark, the study will terminate early if at least 24 patients have been included and no additional patients have been included 2 months in a row.

All exercise tests and experimental procedures will be carried out at the Centre for Physical Activity Research (CFAS) and the Department of Cardiology, Rigshospitalet, Denmark. 
Table 1 Overview of inclusion and exclusion criteria

\section{Eligibility of study participants}

Inclusion criteria

- Age $\geq 40$ years.

- A laboratory-confirmed initial positive test followed by one negative test of SARS-CoV-2 $\leq 6$ months after hospital discharge.

- $\leq 10 \mathrm{~L}$ oxygen requirement during hospitalisation.

\section{Exclusion criteria}

- Present atrial fibrillation/flutter.

- Diagnosed with acute myocarditis.

- Health conditions that prevent participation in the exercise training intervention.

- Patients who cannot undergo magnetic resonance scans (eg, kidney disease or metallic implants).

- Treatment with interleukin 6 receptor antagonists (Tocilizumab, Kevzara) within the last month due to drug interference with cardiopulmonary exercise adaptations.

\section{Recruitment}

Patients $(n=40)$ will be identified and/or recruited by one of the infectious disease consultants employed at the collaborating hospitals where the patient is admitted for COVID-19. We aim to include a minimum of three patients each month. Recruitment will be conducted by the following two methods:

- On discharge the study investigator will inform the patient about the study and thereby recruit him/her as a participant.

- Treatment-responsible physicians will provide the study investigators with a list of eligible COVID-19 survivors discharged within the last 6 months.

Inclusion and exclusion criteria are detailed in table 1. All participants will be given a thorough overview of the study via phone interviews. If eligible after the phone interview, the participant will provide informed oral and written consent. All participants will be informed that they can withdraw from the study at any time for any reason without consequence.

Patients will be invited for baseline visits and will be subsequently randomly allocated into one group receiving standard care (control group) or a group performing supervised HIIT three times a week (as recommended by the American College of Sports Medicine) ${ }^{29}$ (figure 1) over a period of 12 weeks. The baseline measurements include a medical health interview and examination (eg, blood pressure, heart rate, ECG), a $\mathrm{VO}_{2}$ max test, a whole-body dual-energy X-ray absorptiometry (DXA) scan, pulmonary function testing (dynamic spirometry, whole-body plethysmography, diffusing capacity), blood samples, echocardiography, 1 repetition max (RM) chest press and leg press (1RM), oral glucose tolerance test (OGTT), continuous glucose monitoring, axial accelerometer-based physical activity monitors (AX3), cardiac MRI (cMRI) and questionnaires (Post-COVID-19 Functional Status (PFAS) (figure 2), King's Brief Interstitial Lung Disease (KBILD) questionnaire and The Short Form 36 (SF-36) Health Survey. The same measurements will be conducted at the final visit post intervention (figure 1).

\section{Intervention}

The HIIT intervention consists of 12 weeks of HIIT on an ergometer bike three times a week for $38 \mathrm{~min}$. The specific HIIT training protocol is chosen from an initial fidelity and tolerability study which compares intervals of either $4 \times 4$ min, $6 \times 1$ min or $10-20-30$ s (see online supplemental table S1 and online supplemental figures S1 and S2 for details). The HIIT protocol with the highest composite fidelity, tolerability and safety score (see online supplemental table S2) will be used in this study, and in case of

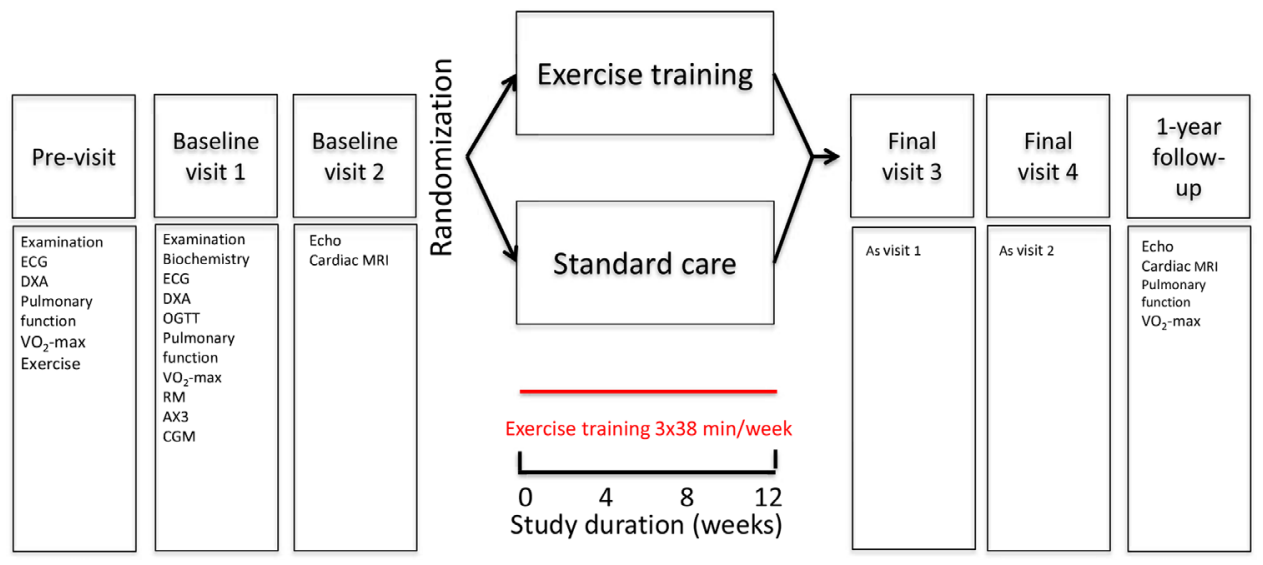

Figure 1 Study overview and tests. AX3, axial accelerometer-based physical activity monitor; CGM, continuous glucose monitoring; DXA, dual-energy X-ray absorptiometry; echo, echocardiography; examination, health examination; OGTT, oral glucose tolerance test; $\mathrm{RM}, 1$ repetition $\max ; \mathrm{VO}_{2} \max$, maximal oxygen consumption. 


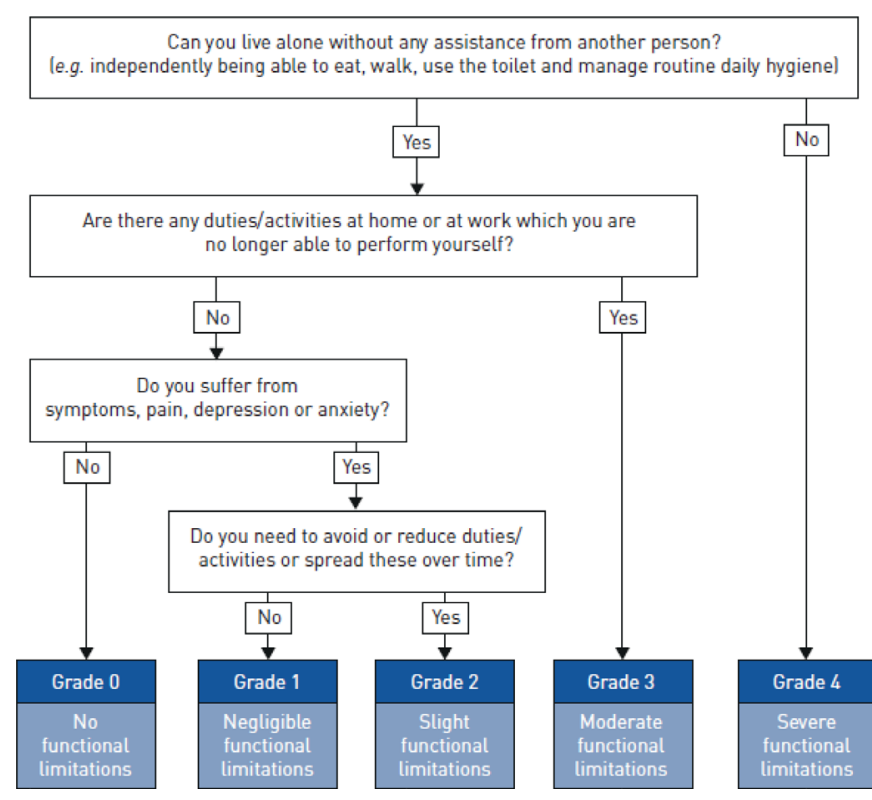

Figure 2 Flow chart of patient self-report method for the Post-COVID-19 Functional Status scale.

equal scores the HIIT protocol with the most time $>85 \%$ of maximal heart rate $\left(\mathrm{HR}_{\max }\right)$ will be chosen.

\section{Outcomes}

The primary outcome is change in left ventricular (LV) mass measured by cMRI before and after 12 weeks of the intervention period. Key secondary outcome is right ventricle volume, also measured by cMRI. The secondary outcomes are listed in table 2. Cardiac structural and functional impairments at baseline and after the intervention period will be assessed by cMRI and TTE, and lung function will be assessed by standardised lung function testing as described in the Lung function testing section. Symptoms and quality of life will be assessed by the three questionnaires mentioned above. Furthermore, markers of systemic inflammation, lung function and metabolic dysfunction, including glucose homeostasis, will be assessed prior to and after the intervention period. The outcomes are assessed as outlined in figure 1 .

\section{Cardiac MRI}

A $1.5 \mathrm{~T}$ whole-body scanner will be used for cMRI. Balanced steady-state free precession cine images will be obtained in three long axes and in a stack of short axes covering the entire heart to rule out wall motion abnormalities and quantify cardiac chamber volumes and ventricular mass. Late gadolinium enhancement imaging and tissue characterisation with parametric mapping will be performed. Gradient echo phase-contrast cine MRI with ECG gating will be used to evaluate aortic and pulmonary artery flows and areas. Images of the ascending and descending aorta will be acquired in the transverse plane perpendicular to the aortic lumen at the level of the right pulmonary artery. Images of the pulmonary arteries will be acquired in two planes perpendicular to the main pulmonary artery and left pulmonary artery, respectively.
Thoracic and abdominal fat will be imaged by a volumetric interpolated breath-hold examination using twopoint Dixon fat-water separation covering the entire thorax and abdomen in the axial plane. A mid-thigh cross-sectional slice will be obtained to measure muscle adaptation before and after the training intervention. Semiautomatic evaluation using commercially available software (cvi42, Circle Cardiovascular Imaging, Calgary, Canada) will be applied for postprocessing. Following automatic contour detection of the LV endocardium and epicardium, all borders will be corrected manually, if necessary, and parameters including LV ejection fraction, stroke volume, end-diastolic volume, end-systolic volume and myocardial mass will be derived accordingly to current guidelines. ${ }^{30-32}$

\section{Transthoracic echocardiography}

TTE will be used to assess cardiac changes and performed with General Electric, GE Vivid E95 (GE Vingmed Ultrasound, Horten, Norway). Offline analyses will be performed using the General Electric EchoPac software. The echocardiograms will be performed and interpreted by one of the study clinicians and validated by a cardiologist blinded to patient data. Chamber quantification will be done according to the recommendations of the European Association of Echocardiography and the American Society of Echocardiography. ${ }^{33}$ LV wall thicknesses will be determined in parasternal long-axis view in end diastole immediately below the mitral valve leaflets. LV mass will be calculated using the following formula: $\mathrm{LV}$ mass $=0.8 \times\{1.04[(\mathrm{LV}$ internal diameter + posterior wall thickness + septal wall thickness $)^{3}-(\mathrm{LV}$ internal diameter) $\left.\left.{ }^{3}\right]\right\}+0.6 \mathrm{~g}$. LV ejection fraction will be determined by Simpson's biplane method. Left atrial volume will be determined by the recommended biplane area-length method and indexed for body surface area. Pulsed-wave Doppler will be performed in the apical fourchamber view, with the sample volume placed between the mitral leaflet tips to obtain diastolic mitral early (E) and atrial (A) inflow velocities and deceleration time of the E velocity. Pulsed-wave early diastolic tissue Doppler velocities (e') will be determined from the apical fourchamber view at the septal and lateral regions of the mitral annulus. ${ }^{34}$ Right ventricular function will be assessed using tricuspid annular plane systolic excursion. Global LV longitudinal strain will be measured using two-dimensional, frame-to-frame, speckle-tracking echocardiography to determine deformation of the left ventricle and has been described in detail elsewhere. ${ }^{35}$ The tracking algorithm follows the myocardium from frame to frame throughout the cardiac cycle in all three apical views. The algorithm allows for a manual adjustment of the region of interest to ensure that all myocardial regions are included and tracked accurately, and accurate tracking is ensured by visual inspection of tracking and segmental strain curves. Finally, a global score is calculated by the algorithm. 
Table 2 Outcome domains and measures assessed at baseline and follow-up (12 weeks after baseline)

\begin{tabular}{|c|c|c|}
\hline Outcome & Domain & Measurement \\
\hline Primary & Change in left ventricular mass. & MRI. \\
\hline Key secondary & Change in RV volume. & MRI. \\
\hline \multirow{9}{*}{ Secondary } & $\begin{array}{l}\text { Functional cardiac parameters: LVEF, global longitudinal strain, E/A } \\
\text { ratio, E', RV volumes, RVEF, TAPSE, change in maximal tricuspid } \\
\text { regurgitation velocity and pressure gradient, RV s', peak E velocity, } \\
\text { peak A velocity, septal e', lateral e', E/e' septal and E/e' lateral. }\end{array}$ & MRI and echocardiography. \\
\hline & $\begin{array}{l}\text { Cardiac inflammation, vascular dysfunction, extracellular volume } \\
\text { and diffuse fibrotic changes. }\end{array}$ & Gadolinium and MRI scan. \\
\hline & Muscle morphology and function. & MRI. \\
\hline & Body composition analysis. & BMI and DXA. \\
\hline & Oral glucose tolerance test. & OGTT. \\
\hline & Continuous glucose monitoring. & CGM. \\
\hline & Axial accelerometer-based physical activity monitors. & AX3. \\
\hline & $\begin{array}{l}\text { Blood samples analysed for markers related to exercise, } \\
\text { metabolism, inflammation and cardiovascular function. }\end{array}$ & \\
\hline & $\begin{array}{l}\text { Questionnaires on quality of life, SF-36, King's Brief Interstitial Lung } \\
\text { Disease questionnaire, Post-COVID-19 Functional Status, Fatigue } \\
\text { Assessment Scale. }\end{array}$ & $\begin{array}{l}\text { SF-36, PCFS, Fatigue Assessment } \\
\text { Scale. }\end{array}$ \\
\hline
\end{tabular}

${ }^{*}$ Training evaluation will be assessed every training.

BMI, body mass index; CGM, continuous glucose monitoring; DXA, dual-energy X-ray absorptiometry; IVS, interventricular septal; LAVI, left atrial volume index; LVEF, left ventricular ejection fraction; LVID, left ventricular internal diameters; OGTT, oral glucose tolerance test; PCFS, Post-COVID-19 Functional Status; PWT, posterior wall thickness; RM, repetition maximum; RPE, rate of perceived exertion; RV, right ventricular; RVEF, right ventricular ejection fraction; SF-36, Short Form 36 Health Survey; TAPSE, tricuspid annular plane systolic excursion; $\mathrm{VO}_{2}$ max, maximal oxygen consumption.

\section{Blood samples}

Following an overnight fast (10 hours) blood samples will be collected and processed by a trained laboratory technician and analysed according to standard procedures. Plasma is stored at $-80^{\circ} \mathrm{C}$ prior to analysis. Blood samples will be analysed for (1) markers of low-grade inflammation, including high-sensitivity $\mathrm{C}$ reactive protein, tumour necrosis factor- $\alpha$, interleukin 1 recepter antagonist (IL-1RA), interferon- $\gamma$ and interleukins (IL-6, IL-10 and others); (2) cardiometabolic biomarkers, including total troponins, D-dimer, creatine kinase, total cholesterol, high-density lipoprotein, low-density lipoprotein, triglycerides, glycated haemoglobin, fasting insulin, fasting plasma glucose and probrain natriuretic peptide; and (3) haematology, electrolytes, and liver and renal status.

\section{Body composition}

DXA will be used to assess total fat mass, bone density and lean body mass at baseline visit and at final visit (Lunar Prodigy, GE Healthcare, Madison, Wisconsin, USA; enCORE V.14.10.022).

\section{Maximal aerobic capacity}

Graded exercise tests will be performed on a bicycle ergometer (Monark LC4, Monark Exercise, Vansbro, Sweden) to determine the maximal oxygen uptake. The test will start with a 5 min warm-up at 50 watts for men and at 30 watts for women. Warm-up is immediately followed by a 20-watt increase every minute until exhaustion, which is categorised as voluntary exhaustion or failure to maintain rounds per minute above 60 . Ventilation rate and expired oxygen and carbon dioxide levels will be measured during the test via an indirect calorimetric 


\section{Fatigue Assessment Scale (FAS)}

The following ten statements refer to how you usually feel. Per statement you can choose one out of five answer categories, varying from Never to Always.

Please circle the answer to each question that is applicable to you. Please give an answer to each question, even if you do not have any complaints at the moment.

1 = Never, 2 = Sometimes (about monthly or less); 3 = Regularly (about a few times a month); 4 = Often (about weekly) and 5 = Always (about every day).

Never Sometimes Regularly Often Always

\begin{tabular}{|c|c|c|c|c|c|}
\hline 1. I am bothered by fatigue & 1 & 2 & 3 & 4 & 5 \\
\hline 2. I get tired very quickly & 1 & 2 & 3 & 4 & 5 \\
\hline 3. I don't do much during the day & 1 & 2 & 3 & 4 & 5 \\
\hline 4. I have enough energy for everyday life & 1 & 2 & 3 & 4 & 5 \\
\hline 5. Physically, I feel exhausted & 1 & 2 & 3 & 4 & 5 \\
\hline 6. I have problems to start things & 1 & 2 & 3 & 4 & 5 \\
\hline 7. I have problems to think clearly & 1 & 2 & 3 & 4 & 5 \\
\hline 8. I feel no desire to do anything & 1 & 2 & 3 & 4 & 5 \\
\hline 9. Mentally, I feel exhausted & 1 & 2 & 3 & 4 & 5 \\
\hline $\begin{array}{l}\text { 10. When I am doing something, I can } \\
\text { concentrate quite well }\end{array}$ & 1 & 2 & 3 & 4 & 5 \\
\hline
\end{tabular}

Figure 3 A 10-item general fatigue questionnaire for assessing both physical and mental fatigue (Fatigue Assessment Scale).

system, and heart rate will be monitored simultaneously (Quark CPET, COSMED, Rome, Italy).

\section{Lung function testing}

Standardised lung function testing will be performed in accordance with consensus guidelines by dynamic spirometry, ${ }^{36}$ body plethysmography ${ }^{37}$ and single-breath carbon monoxide uptake. ${ }^{38}$ The following parameters will be collected from the lung function tests: forced expiratory volume in $1 \mathrm{~s}$, forced vital capacity, total lung capacity, residual volume and diffusing capacity for carbon monoxide corrected for haemoglobin. Based on summary equations, ${ }^{39} 40$ the expected values according to height, age and sex will be calculated.

\section{Oral glucose tolerance test}

The standard 2-hour 75 g glucose OGTT will be performed following an overnight fast (10 hours). Blood samples will be drawn at baseline (0) and after 60 and $120 \mathrm{~min}$ and analysed for plasma glucose, insulin and $\mathrm{C}$ peptide.

\section{Physical activity level}

Posture and physical activity behaviours are measured using three axial accelerometer-based physical activity monitors (Axivity AX3, Newcastle, UK). All participants will be equipped with two accelerometers for 5 consecutive days, with one accelerometer placed on the right thigh and one accelerometer placed on the right side of the lower back. Both accelerometers will be attached with a patch (Fixomull Stretch, BSN Medical, Germany). The participants will be instructed to register what time they get up in the morning and go to bed in the evening, time of potential working hours, and time for resistance or endurance exercise, and given specific written instructions on how to register and what to do if the AX3 falls off accidentally. Using the AX3 to monitor allocation and basic physical activity behaviour will take place before, during and after the intervention period.

\section{Enjoyment, tolerance and verification of training intensity during HIIT}

Enjoyment and perceived tolerance as well as perceived exertion will be evaluated during each HIIT session. The 10-point Likert scale evaluates enjoyment and perceived tolerance of each HIIT protocol at the end of each training session. ${ }^{41}$ Patients have to indicate the degree to which they agreed with the following statement before training, 'How motivated are you for today's training?', and the following two statements after training: 'How enjoyable have today's training session been?' and 'How tolerant has today's training been?' Verification of training intensity is evaluated after completed training (1-10 Borg scale) $)^{42}$ and from accumulated minutes above $85 \%$ of $\mathrm{HR}_{\max }$. Should we measure a heart rate higher than the previous $\mathrm{HR}_{\max }$ during a training session, the $\mathrm{HR}_{\max }$ will be updated from next training session.

\section{Symptoms and quality of life}

Symptoms and quality of life will be assessed by the PFAS $^{43}$ (figure 2), Fatigue Assessment Scale (FAS) (figure 3), ${ }^{44}$ KBILD questionnaire ${ }^{45}$ and SF-36 Health Survey $^{46}$ (table 2) at baseline and at follow-up. PFAS is a tool to measure the full spectrum of functional outcomes following COVID-19 and is used to measure the morbidity and persisting symptoms following COVID-19. It is a scale from grade 0 to grade 4 , where grade 0 reflects no functional limitations, whereas grade 1 upwards reflect symptoms, pain and anxiety to an increasing degree. ${ }^{43}$ The 10-item FAS is used to measure fatigue during everyday life. ${ }^{44}$ The KBILD questionnaire is a self-completed measure of health status and consists of 15 items with a 7-point Likert response scale with three domains: psychological, breathlessness and activities, and chest symptoms, combined in a total score. The total score ranges from 0 to 100 , with 100 representing the best health status. The questionnaire is normally used in patients with interstitial lung disease ${ }^{45}$. The SF-36 questionnaire is a self-administered questionnaire containing 36 items. It measures general health on eight multi-item dimensions, covering functional status, well-being and overall evaluation of health ${ }^{46}$.

\section{Safety}

At baseline visit the participant will undergo a medical health interview and examination by a physician to assess health status. Each time a participant is to engage in an exercise training session, the following contraindications 
and precautions will be considered: acute or recurring chest pain or systolic blood pressure $>200 \mathrm{~mm} \mathrm{Hg} \pm$ diastolic blood pressure $>100 \mathrm{~mm} \mathrm{Hg}$. Furthermore, the following termination criteria will be used during an exercise training session: onset of angina or angina-like symptoms, signs of poor perfusion (eg, confusion, nausea, light-headedness, central cyanosis, requests to terminate (eg, due to intolerable dyspnoea), and physical or verbal manifestations of severe fatigue).

\section{Risk of adverse events during the study}

Intravenous contrast will be administered during cMRI scans as normal clinical routine. The amount of blood drawn will be $100 \mathrm{~mL}$; therefore, the risk of dizziness, dyspnoea, or other symptoms of anaemia or hypovolaemia is minimal. In addition, the blood samples are drawn according to standard procedures, making the risk of infection minimal. Besides these, only non-invasive examinations will be performed throughout the study. There are no known harms of cMRI or echocardiography since the imaging techniques are magnetic resonance and ultrasound, respectively. Intravenous contrast administered during cMRI can rarely cause acute allergic reactions. Experienced physicians will be present and can manage such situations. Maximal aerobic capacity and RM measurements can cause some degree of exhaustion, but this is a routine method for scientific purposes in the laboratory of CFAS.

DXA scans involves radiation corresponding to approximately one-tenth of the radiation acquired by a thoracic X-ray $(0.01 \mathrm{mSv})$. This correlates to $0.03 \%$ of the received annual background radiation $(3 \mathrm{mSv})$. The DXA scan is therefore generally considered safe and it is part of a routine scientific method at CFAS. OGTT does not cause any discomforts or risks, and after completion the participants will be offered a light meal.

A small peripheral venous catheter (PVC) will be inserted in the antecubital fossa of the patient in accordance with standard procedures due to the repetitive blood sample drawing of these tests. Insertion of a PVC

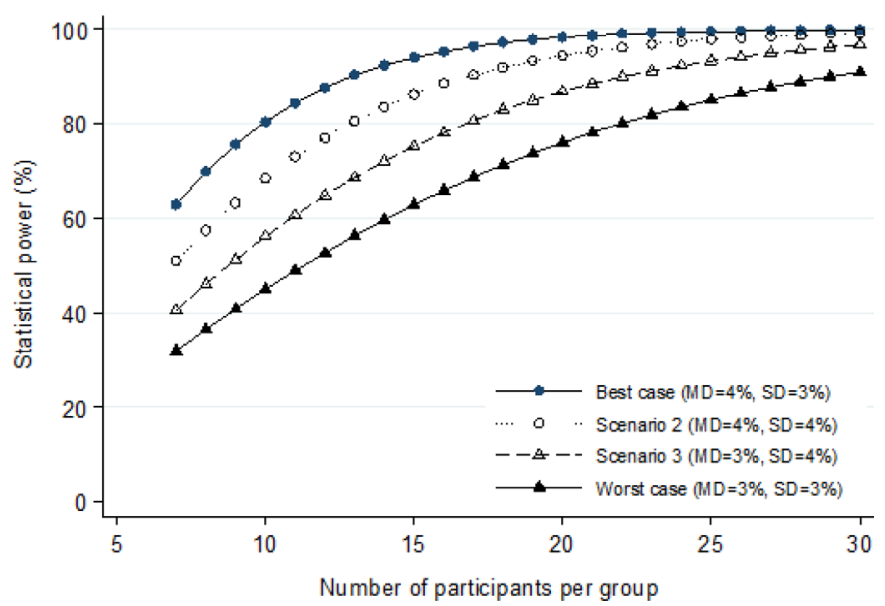

Figure 4 Sample size scenarios. MD, mean difference of change between the intervention groups. poses a small risk of infection (eg, thrombophlebitis) and can potentially cause bruising. The patients will be informed about this and instructed to contact us in case of symptoms of infection.

Lung function testing may be associated with some degree of exhaustion and discomfort but is routinely used in clinical practice and is considered a low-risk procedure.

Adverse events will be reported at baseline visit, every fourth week during the intervention (both in the HIIT group and in the standard care group) and at final visit. The following events will be recorded: signs of infections, hospital admissions, angina pectoris and injuries/ pain that prevent participation in training. Any serious adverse events (death, life-threatening, hospital admission, expected as well as unexpected) will be reported to the Regional Ethical Committee of the Capital Region of Denmark within 7 days after the study principal investigator or physician has become aware of the occurrence. ${ }^{47}$

\section{Data collection and management}

The study coordinator and cardiac magnetic resonance imaging personnel will be responsible for managing patient data collection. Research staff will enter data into a secure web-based database (EasyTrial). Access is password-protected and limited to study personnel. Only anonymised data will be recorded. All data will be stored on a secure server and paper copies will be maintained in an appropriately locked and secured cabinet. To promote patient retention and completion of follow-up assessments, patients in the standard care group will receive a personal phone call with the study coordinator once a month, where activity level, general health, hospital admission and infections since baseline visit will be noted.

\section{Statistical procedures}

\section{Sample size}

Inspired by a study ${ }^{48}$ performed at our research centre, it is expected that the HIIT group will increase left ventricular mass (LVM) at least $4 \%$ more than the standard care group with an SD of 3\%. For a contrast analysis of covariance (ANCOVA) with two means and a correlation of 0.85 between the baseline and follow-up values, using a two-sided significance level of $0.05,15$ persons in each group is required to obtain a statistical power $(1-\beta)$ of $80 \%$. However, as the target group for this study is expected to be more heterogeneous compared with the previous study, we assume a lower precision of the estimate. Moreover, we will operate with a statistical power of $90 \%$ to account for loss to follow-up. Thus, with an assumed SD of 4\%, 20 participants in each group (40 in total) will be included in the study to obtain a statistical power of $90 \%$ (scenario 2 in figure 4 ). The different sample size scenarios are depicted in figure 4 .

\section{Analysis population}

The study follows an intention-to-treat (ITT) principle with use of baseline observations carried forward imputation, where the baseline value will replace missing 
data. ${ }^{49}$ The primary analysis will include the data as observed, and the imputation technique will be used in the sensitivity analyses to assess the robustness of the results. The ITT principle is chosen to avoid bias since non-adherers versus adherers generally differ regarding motivational and prognostic factors. Patterns of non-adherence and other missing data will be investigated. Satisfactory adherence is defined as a minimum of $\geq 80 \%$ adherence to the prescribed HIIT sessions. Any discrepancies in data analysis between the methods will be resolved by a third-party biostatistician.

\section{Statistical analysis plan and reporting}

Continuous outcomes will be compared by ANCOVA modelling with two-fixed factors (group and time) and baseline value as a covariate. Least squares-means adjusted for baseline variables. To assess the adequacy of the linear models describing the observed data-and to check the assumptions for the systematic and the random parts of the models-we will investigate the model features via the predicted values and the residuals. Hence, the residuals must be normally distributed (around 0 ) and be independent of the predicted values. The change in cMRI-based LV mass will be the primary response variable, and the baseline value (one for each participant), intervention group (two levels) and time (two levels) will be included as covariates.

Categorical outcomes will be analysed by logistic regression.

Outcomes will not be analysed before the end of the study. The results will be reported in accordance with the Consolidated Standards of Reporting Trials. ${ }^{50} 51$

All reported $\mathrm{p}$ values will be two-sided and will not be adjusted for multiple comparisons. Per default, we set the statistical significance at the conventional level of $\mathrm{p}<0.05$. All analyses will be performed using commercially available statistical software.

\section{Randomisation: sequence generation, allocation concealment and} blinding

The randomisation procedure involves a computergenerated block randomisation schedule (Research Randomizer V.4) where patients are randomised in a ratio of 1:1 stratified by sex to an HIIT group or a standard care control group. To ascertain concealment, the block sizes will not be disclosed. The randomised sequence generation will be generated by a researcher who is not involved in the testing. The generated sequence will be forwarded to another researcher not involved in any study procedures and stored on a password-protected computer. Sequentially numbered (according to the sequence) opaque, sealed envelopes will be prepared and stored in a locked cabinet. The envelopes will be lined with aluminium foil to render the envelope impermeable to intense light. Following the conclusion of the baseline examination, the appropriate envelope will be opened by a study nurse and the participant will be informed about the group allocation.
Assessors of imaging-based outcomes will be blinded to patient data, including allocation at baseline and follow-up. Owing to the nature of the study, patients cannot be blinded to the exercise training modality.

\section{Patient and public involvement}

Neither patients nor the public were involved in the writing of this protocol and will not formally be involved in the dissemination of the findings. The patients will receive their test results from the baseline and follow-up post intervention/standard care.

\section{Strengths and limitations}

This is the first randomised study to examine the safety and effects of a supervised HIIT intervention on cardiac structure and function in previously hospitalised patients with COVID-19 and our data will hopefully contribute to a better understanding of long COVID-19 and knowledge to whether HIIT has an impact on morbidity and mortality in COVID-19 survivors. The 12-week HIIT intervention used in this study consists of three-weekly session of $38 \mathrm{~min}$ and has previously shown to mitigate comorbidities that are like those seen in COVID-19 survivors. Another strength is the stateof-art method to assess changes in cardiopulmonary function and exercise capacity.

Despite these strengths, there are some limitations to this study as well. We lack previous data before COVID-19 infection; henceforth, the baseline prior to infection is unknown, which makes it difficult to conclude whether abnormalities in cardiac structure and function are COVID-19-related. Only previously hospitalised patients were included in this study, which limits the generalisability of the study and introduces a possible selection bias. The patients are included at different stages of recovery (0-6 months after hospital discharge), which makes the group more heterogeneous, where the impact of the intervention possibly will be different for patients enrolled at discharge close versus further away from their individual discharge. Although we expect the intervention to have an effect in the same direction for these patients, we aim to include patients as close to discharge as possible to limit this heterogeneity.

\section{Ethics statement}

The research ethics committee of the Capital Region of Denmark approved the study before study initiation (H-20033733 with amendments 75068 and 75799). The study will be conducted in accordance with the Helsinki Declaration. Written and oral consent will be obtained from all participants prior to enrolment in the study. The manuscript based on these findings will be submitted to peer-reviewed journals, including cases of positive, negative and inconclusive results, targeting open-access journals. Prior to submission, the initial manuscript and the original data will be uploaded to a public repository. 


\section{CONCLUSIONS}

We anticipate that the results obtained by this study will inform future guidelines on exercise training rehabilitation of previously hospitalised COVID-19 patients.

\section{Author affiliations}

${ }^{1}$ Centre for Physical Activity Research (CFAS), Copenhagen University Hospital Rigshospitalet, Copenhagen, Denmark

${ }^{2}$ Department of Biomedical Sciences, Faculty of Health and Medical Sciences, University of Copenhagen, Copenhagen, Denmark

${ }^{3}$ Department of Infectious Diseases, Copenhagen University Hospital - Hvidovre Hospital, Hvidovre, Denmark

${ }^{4}$ Department of Pulmonary Medicine and Infectious Diseases, Copenhagen University Hospital - North Zealand Hospital, Hillerød, Denmark

${ }^{5}$ Department of Cardiology, University Hospital Copenhagen - Rigshospitalet, Copenhagen, Denmark

${ }^{6}$ Neurovascular Research Laboratory, Faculty of Life Sciences and Education, University of South Wales, Pontypridd, UK

${ }^{7}$ Department of Clinical Physiology and Nuclear Medicine, University Hospital Copenhagen - Rigshospitalet, Copenhagen, Denmark

\section{Twitter Ronan M G Berg @CRESP_Research}

Contributors RHC and RMGB conceived the study. FF, IER, JBB, RHC and RMGB initially designed the study. MR-L and VR elaborated on the HIIT intervention and the statistical design of the study. RSR and SJ contributed to the design and conduct of the study. PGJ, LK, MAVL and NV designed the echocardiographic and CMRI protocols. FF and IER wrote the first manuscript draft. All authors made critical revisions and approved the final manuscript.

Funding CFAS is supported by TrygFonden grants (ID 101390, ID 20045 and ID 125132).

Competing interests MR-L has received personal lecture fees from Novo Nordisk A/S. PGJ reports honorarium from Novo Nordisk and AstraZeneca. LK reports speaker honorarium from Novo, Novartis, Boehringer and AstraZeneca, unrelated to this manuscript.

Patient consent for publication Not required.

Provenance and peer review Not commissioned; externally peer reviewed.

Supplemental material This content has been supplied by the author(s). It has not been vetted by BMJ Publishing Group Limited (BMJ) and may not have been peer-reviewed. Any opinions or recommendations discussed are solely those of the author(s) and are not endorsed by BMJ. BMJ disclaims all liability and responsibility arising from any reliance placed on the content. Where the content includes any translated material, BMJ does not warrant the accuracy and reliability of the translations (including but not limited to local regulations, clinical guidelines, terminology, drug names and drug dosages), and is not responsible for any error and/or omissions arising from translation and adaptation or otherwise.

Open access This is an open access article distributed in accordance with the Creative Commons Attribution Non Commercial (CC BY-NC 4.0) license, which permits others to distribute, remix, adapt, build upon this work non-commercially, and license their derivative works on different terms, provided the original work is properly cited, appropriate credit is given, any changes made indicated, and the use is non-commercial. See: http://creativecommons.org/licenses/by-nc/4.0/.

\section{ORCID iDs}

Iben Elmerdahl Rasmussen http://orcid.org/0000-0001-6349-0308 Rasmus Syberg Rasmussen http://orcid.org/0000-0002-5892-7859 Ronan M G Berg http://orcid.org/0000-0002-5757-9506

\section{REFERENCES}

1 Shi S, Qin M, Shen B, et al. Association of cardiac injury with mortality in hospitalized patients with COVID-19 in Wuhan, China. JAMA Cardiol 2020;5:802-8.

2 Madjid M, Safavi-Naeini P, Solomon SD, et al. Potential effects of coronaviruses on the cardiovascular system: a review. JAMA Cardiol 2020;5:831-10.

3 Xu Z, Shi L, Wang Y. Pathological findings of COVID-19 associated with acute respiratory distress syndrome. Lancet Respir Med 2020;2600:19-21.
4 Puntmann VO, Carerj ML, Wieters I, et al. Outcomes of cardiovascular magnetic resonance imaging in patients recently recovered from coronavirus disease 2019 (COVID-19). JAMA Cardiol 2020;5:1265-73.

5 Bonow RO, Fonarow GC, O'Gara PT, et al. Association of coronavirus disease 2019 (COVID-19) with myocardial injury and mortality. JAMA Cardiol 2020;5:751.

6 Danzi GB, Loffi M, Galeazzi G, et al. Acute pulmonary embolism and COVID-19 pneumonia: a random association? Eur Heart $J$ 2020;41:1858

7 Rooney S, Webster A, Paul L. Systematic review of changes and recovery in physical function and fitness after severe acute respiratory syndrome-related coronavirus infection: implications for COVID-19 rehabilitation. Phys Ther 2020;100:1717-29.

8 Rees EM, Nightingale ES, Jafari Y, et al. COVID-19 length of hospital stay: a systematic review and data synthesis. BMC Med 2020;18:270.

9 Olsen $\mathrm{RH}$, Krogh-Madsen $\mathrm{R}$, Thomsen $\mathrm{C}$, et al. Metabolic responses to reduced daily steps in healthy nonexercising men. JAMA 2008;299:1261-3.

10 Nielsen ST, Harder-Lauridsen NM, Benatti FB, et al. The effect of 8 days of strict bed rest on the incretin effect in healthy volunteers. $J$ Appl Physiol 2016;120:608-14.

11 Vis JC, de Bruin-Bon RH, Bouma BJ, et al. 'The sedentary heart': physical inactivity is associated with cardiac atrophy in adults with an intellectual disability. Int J Cardiol 2012;158:387-93.

12 Scott JM, Martin D, Ploutz-Snyder R, et al. Efficacy of exercise and testosterone to mitigate atrophic cardiovascular remodeling. Med Sci Sports Exerc 2018;50:1940-9.

13 Ried-Larsen M, Aarts HM, Joyner MJ. Effects of strict prolonged bed rest on cardiorespiratory fitness: systematic review and metaanalysis. J Appl Physiol 2017;123:790-9.

14 Carfi A, Bernabei R, Landi F, et al. Persistent symptoms in patients after acute COVID-19. JAMA 2020;324:603-5.

15 Rubin R. As their numbers grow, COVID-19 "long haulers" stump experts. JAMA 2020;324:1381-3.

16 Ngai JC, Ko FW, Ng SS, et al. The long-term impact of severe acute respiratory syndrome on pulmonary function, exercise capacity and health status. Respirology 2010;15:543-50.

17 Arbab-Zadeh A, Perhonen M, Howden E, et al. Cardiac remodeling in response to 1 year of intensive endurance training. Circulation 2014;130:2152-61.

18 Nigro E, Polito R, Alfieri A. Molecular mechanisms involved in the positive effects of physical activity on coping with COVID-19. Eur J Appl Physiol 2020;120:2582.

19 Lau HM-C, Ng GY-F, Jones AY-M, et al. A randomised controlled trial of the effectiveness of an exercise training program in patients recovering from severe acute respiratory syndrome. Aust $J$ Physiother 2005;51:213-9.

20 Hermann M, Pekacka-Elgi A-M, Witassek F. Feasibility and efficacy of cardiopulmonary rehabilitation following COVID-19. Am J Phys Med 2020.

21 Fernandes T, Baraúna VG, Negrão CE, et al. Aerobic exercise training promotes physiological cardiac remodeling involving a set of microRNAs. Am J Physiol Heart Circ Physiol 2015;309:H543-52.

22 Lavie CJ, Milani RV, Marks P, et al. Exercise and the heart: risks, benefits, and recommendations for providing exercise prescriptions. Ochsner J 2001;3:207-12.

23 Why Doesn't Exercise Grow the Lungs When Other Factors Do? Exercise and Sport Sciences Reviews.

24 Keech A, Holgate K, Fildes J, et al. High-intensity interval training for patients with coronary artery disease: finding the optimal balance. Int J Cardiol 2020;298:8-14.

25 Batacan RB, Duncan MJ, Dalbo VJ, et al. Effects of high-intensity interval training on cardiometabolic health: a systematic review and meta-analysis of intervention studies. $\mathrm{Br} J$ Sports Med 2017;51:494-503.

26 Karlsen T, Aamot I-L, Haykowsky M, et al. High intensity interval training for maximizing health outcomes. Prog Cardiovasc Dis 2017;60:67-77.

27 Mo X, Jian W, Su Z, et al. Abnormal pulmonary function in COVID-19 patients at time of hospital discharge. Eur Respir J 2020;55. doi:10.1183/13993003.01217-2020. [Epub ahead of print: 1806 2020].

28 Andrianopoulos V, Celli BR, Franssen FME, et al. Determinants of exercise-induced oxygen desaturation including pulmonary emphysema in COPD: results from the eclipse study. Respir Med 2016;119:87-95.

29 Physical activity guidelines resources. Available: https://www.acsm. org/read-research/trending-topics-resource-pages/physical-activityguidelines [Accessed 16 Nov 2020]. 
30 Schulz-Menger J, Bluemke DA, Bremerich J, et al. Standardized image interpretation and post processing in cardiovascular magnetic resonance: society for cardiovascular magnetic resonance (SCMR) board of trustees task force on standardized post processing. $J$ Cardiovasc Magn Reson 2013;15:35.

31 Friedrich MG, Marcotte F. Cardiac magnetic resonance assessment of myocarditis. Circ Cardiovasc Imaging 2013;6:833-9.

32 Thackeray JT, Bengel FM. Molecular imaging of myocardial inflammation with positron emission tomography post-ischemia: a determinant of subsequent remodeling or recovery. JACC CardiovasC Imaging 2018;11:1340-55.

33 Lang RM, Badano LP, Mor-Avi V, et al. Recommendations for cardiac chamber quantification by echocardiography in adults: an update from the American Society of echocardiography and the European association of cardiovascular imaging. J Am Soc Echocardiogr 2015;28:e14:1-39.

34 Nagueh SF, Smiseth OA, Appleton CP, et al. Recommendations for the Evaluation of Left Ventricular Diastolic Function by Echocardiography: An Update from the American Society of Echocardiography and the European Association of Cardiovascular Imaging. J Am Soc Echocardiogr 2016;29:277-314.

35 Jensen MT, Sogaard P, Andersen HU, et al. Global longitudinal strain is not impaired in type 1 diabetes patients without albuminuria: the Thousand \& 1 study. JACC Cardiovasc Imaging 2015;8:400-10.

36 Miller MR, Hankinson J, Brusasco V, et al. Standardisation of spirometry. Eur Respir J 2005;26:319-38.

37 Wanger J, Clausen JL, Coates A, et al. Standardisation of the measurement of lung volumes. Eur Respir J 2005;26:511-22.

38 Macintyre N, Crapo RO, Viegi G, et al. Standardisation of the singlebreath determination of carbon monoxide uptake in the lung. Eur Respir J 2005;26:720-35.

39 Quanjer PH, Tammeling GJ, Cotes JE, et al. Lung volumes and forced ventilatory flows. Eur Respir J 1993;6 Suppl 16:5-40.
40 Cotes JE, Chinn DJ, Quanjer PH, et al. Standardization of the measurement of transfer factor (diffusing capacity). Eur Respir $J$ 1993;6 Suppl 16:41-52.

41 Afthanorhan A. The Likert scale analysis using parametric based structural equation modeling (SEM). Comput Methods Soc Sci 2016;4:13-21.

42 Borg GAV. Psychophysical bases of perceived exertion. Plast Reconstr Surg 1954:377-81.

43 Klok FA, Boon GJAM, Barco S, et al. The Post-COVID-19 functional status scale: a tool to measure functional status over time after COVID-19. Eur Respir J 2020;56. doi:10.1183/13993003.014942020. [Epub ahead of print: 0207 2020].

44 Michielsen HJ, De Vries J, Van Heck GL. Psychometric qualities of a brief self-rated fatigue measure: the fatigue assessment scale. $J$ Psychosom Res 2003;54:345-52.

45 Prior TS, Hilberg O, Shaker SB, et al. Validation of the king's brief interstitial lung disease questionnaire in idiopathic pulmonary fibrosis. BMC Pulm Med 2019;19:255.

46 Brazier JE, Harper R, Jones NM, et al. Validating the SF-36 health survey questionnaire: new outcome measure for primary care. BMJ 1992;305:160-4.

47 When to report side effects (adverse reactions)? Available: www.nvk. dk [Accessed 10 Nov 2020].

48 Christensen RH, Wedell-Neergaard A-S, Lehrskov LL, et al. Effect of aerobic and resistance exercise on cardiac adipose tissues: secondary analyses from a randomized clinical trial. JAMA Cardiol 2019;4:778-87.

49 Ware $\mathrm{JH}, \mathrm{Ph} \mathrm{D}$. Interpreting incomplete data in studies of diet and weight loss. N Engl J Med 2003;348:2136-7.

50 Moher D, Hopewell S, Schulz KF, et al. CONSORT 2010 explanation and elaboration: updated guidelines for reporting parallel group randomised trials. Int J Surg 2012;10:28-55.

51 Christensen R, Bliddal H, Henriksen M. Enhancing the reporting and transparency of rheumatology research: a guide to reporting guidelines. Arthritis Res Ther 2013;15:109. 
Correction: Protective potential of high-intensity interval

training on cardiac structure and function after COVID-19:

protocol and statistical analysis plan for an investigator-

blinded randomised controlled trial

Rasmussen IE, Foged F, Bjørn Budde J, et al. Protective potential of high-intensity interval training on cardiac structure and function after COVID-19: protocol and statistical analysis plan for an investigator-blinded randomised controlled trial. BMJ Open 2021;11:e048281. doi: 10.1136/bmjopen-2020-048281

The authors want to alert the readers that an error has been noted in the sample size and power calculations. The section 'Sample size' has therefore been rephrased as outlined below, and figure 4 has been modified accordingly. These changes do not affect the design, conduct or the conclusions that can be drawn from the study.

\section{Sample size}

Inspired by a previous study performed at our research centre (Christensen et al. Circulation. 2019 Nov 12;140(20):1684-1686. doi: 10.1161/CIRCULATIONAHA.119.042287; Christensen et al. JAMA Cardiol. 2019 Aug 1;4 (8):778-787. doi: 10.1001/jamacardio.2019.2074), it is expected that the HIIT group will increase left ventricular mass (LVM) up to $17 \%$ more than the standard care group with an SD of $10 \%$. However, as the target group for this study is expected to be more heterogeneous compared with the previous study, we assume a lower effect size and precision of the estimate. The different sample size scenarios are depicted in figure 4.

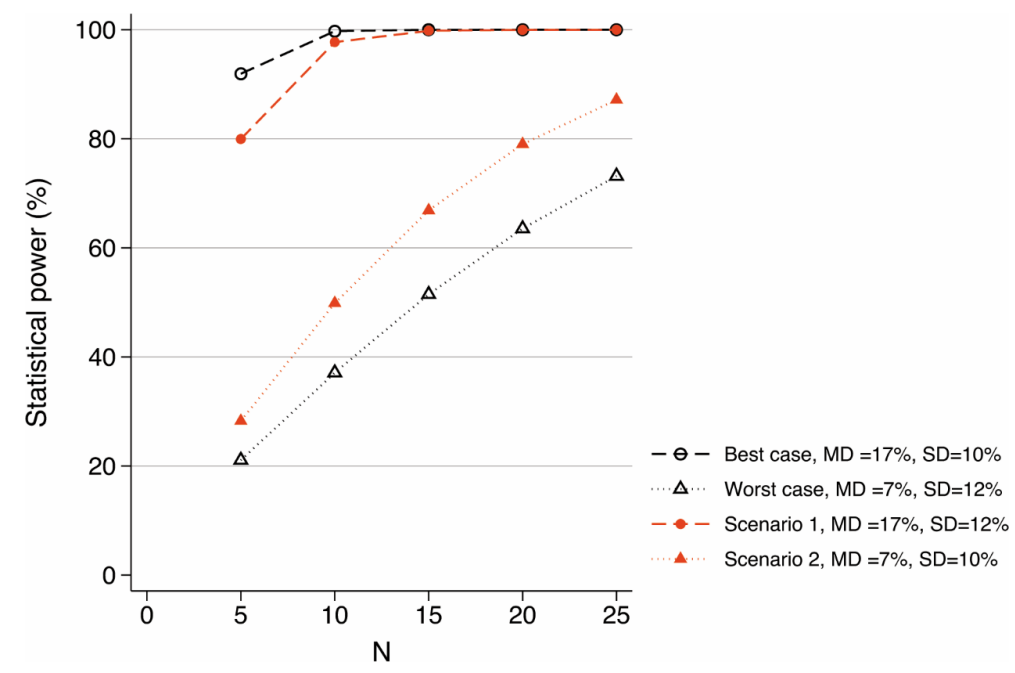

Open access This is an open access article distributed in accordance with the Creative Commons Attribution Non Commercial (CC BY-NC 4.0) license, which permits others to distribute, remix, adapt, build upon this work non-commercially, and license their derivative works on different terms, provided the original work is properly cited, appropriate credit is given, any changes made indicated, and the use is non-commercial. See: http://creativecommons.org/licenses/by-nc/4.0/.

(c) Author(s) (or their employer(s)) 2023. Re-use permitted under CC BY-NC. No commercial re-use. See rights and permissions. Published by BMJ.

BMJ Open 2023;13:e048281corr1. doi:10.1136/bmjopen-2020-048281corr1

Check for updates 